\title{
Optimisation of a tomography sensor for imaging of temperature in a gas turbine engine
}

DOI:

10.1109/ICSENS.2013.6688350

Link to publication record in Manchester Research Explorer

\section{Citation for published version (APA):}

Wood, M. P., \& Ozanyan, K. B. (2013). Optimisation of a tomography sensor for imaging of temperature in a gas turbine engine. In IEEE SENSORS 2013 - Proceedings/IEEE SENSORS - Proc.

https://doi.org/10.1109/ICSENS.2013.6688350

\section{Published in:}

IEEE SENSORS 2013 - Proceedings|IEEE SENSORS - Proc.

\section{Citing this paper}

Please note that where the full-text provided on Manchester Research Explorer is the Author Accepted Manuscript or Proof version this may differ from the final Published version. If citing, it is advised that you check and use the publisher's definitive version.

\section{General rights}

Copyright and moral rights for the publications made accessible in the Research Explorer are retained by the authors and/or other copyright owners and it is a condition of accessing publications that users recognise and abide by the legal requirements associated with these rights.

\section{Takedown policy}

If you believe that this document breaches copyright please refer to the University of Manchester's Takedown Procedures [http://man.ac.uk/04Y6Bo] or contact uml.scholarlycommunications@manchester.ac.uk providing relevant details, so we can investigate your claim.

\section{OPEN ACCESS}




\section{Optimisation of a Tomography Sensor for Imaging of Temperature in a Gas Turbine Engine}

\author{
Michael P. Wood and Krikor B. Ozanyan \\ School of Electrical and Electronic Engineering \\ The University of Manchester \\ Manchester, UK.
}

\begin{abstract}
We present a numerical study of tomographic reconstruction of gas temperature distributions from synthetic data of laser absorption measurements through the imaging plane. A mathematical expression which predicts the reconstruction error as a function of the beam configuration is developed, and a global optimization scheme is employed to minimize this predicted error subject to a constraint on the maximum number of permitted beams. This optimization methodology is applied to an annular measurement region and compared to conventional regular fan-beam arrangements in temperature tomography simulations. Synthetic data generated over the optimized beams led to temperature reconstructions with superior accuracy, provided Tikhonov inversion was used as the method of reconstruction.
\end{abstract}

\section{INTRODUCTION}

The design of a turbine engine involves a compromise between many different parameters: the thermal efficiency of an engine increases with the combustion temperature, but there are strict limits on the temperature of the downstream turbine blades which ensure longevity and safety criteria are met. Striking a balance between these competing demands requires an iterative design and test loop which can be lengthy and expensive, and additional knowledge of internal gas temperature distributions may help in the design of safer and more fuel-efficient engines in the future.

Thermometry in the turbine engine can be achieved using either optical pyrometry of the turbine blades [1, 2], whereby the surface temperature is inferred from thermal emission measurements, or by positioning a thermocouple array downstream of the high-pressure (HP) stages. Neither approach gives information about spatial distributions of temperature upstream of the turbines.

Tunable diode laser absorption spectroscopy offers the unique capability of non-invasive imaging of a cross-sectional slice of the temperature distribution using measurement hardware (such as collimators) that is entirely confined to the imaging plane. This indirect imaging method is well-suited to the severely limited optical access inside a turbine engine, where more conventional temperature imaging techniques such as pyrometry of soot particles [3] or planar laser-induced fluorescence (PLIF) [4], which require an out-of-plane detector array, fail.
Line-of-sight thermometry by laser absorption spectroscopy is commonly achieved by targeting a pair of molecular absorption transitions of an infra-red active molecule $[5,6]$. This approach is effective (if an appropriate line pair is chosen) because absorption transitions from ground states with different energy levels are affected differently by the gas temperature (via the Boltzmann populations). If a ratio is taken of the measured integrated absorbances of the two lines then we obtain a number which is highly sensitive to the gas temperature but relatively insensitive to the species concentration along the beam.

This method has recently been extended to a twodimensional case where laser transmittances over a set of coplanar beams are simultaneously measured. Provided the measured (or derived thereof) quantity varies linearly with a variable in the imaging domain, tomographic reconstruction is feasible. Temperature is not such a variable, but the spectral absorption coefficient $\alpha(x, y, v)$ is, and a tomography problem can be constructed by noticing that the Radon transform of $\alpha$ is relatable to the laser transmittance over a single beam:

$-\ln [\tau(s, \phi ; v)]=$
$\iint_{\Omega} \delta(s-x \cos \phi-y \cos \phi) \alpha(x, y ; v) d x d y$

In this notation, $\tau(s, \phi, v)$ represents the transmittance over a beam, parameterized by $(s, \phi)$ with the equation:

$$
x \cos \phi+y \sin \phi=s
$$

At a frequency $v . \Omega$ represents the measurement domain and $\delta$ is the delta function. Once $\tau(s, \phi ; v)$ is measured over a number of beams and frequencies, the task is to solve equation 1 for $\alpha$.

Once these values of $\alpha(x, y ; v)$ are reconstructed it is possible to infer local values of the gas temperature using HITRAN parameters [7] and a nonlinear least-squares spectral fitting algorithm to solve for the local temperature and species concentration. Our approach (detailed further in [8]) differs from that of low pressure applications [9-12] in that we cannot assume that the gas pressure is low enough for individual absorption features to be distinguishable: this assumption is 
only true for pressures below around 5 bar [13], but commercial turbofan engines often operate at pressure ratios of 40:1 (i.e. 40 bar in the combustor). The spectral fitting approach is a more computationally expensive but ultimately unavoidable necessity if temperature distributions are to be reconstructed within the turbine engine.

\section{TOMOGRAPHIC RECONSTRUCTION}

The task of reconstructing a two-dimensional distribution from its integrals over a limited number of lines is made possible using a method of discretization. The unknown distribution $\alpha(x, y ; v)$ can be approximated by a series expansion whereby it is re-written as a finite sum of weighted basis functions $x_{1}, x_{2}, \ldots$. These basis functions are most often chosen to be flat-topped square pixels for numerical simplicity, and the task is transformed into one of finding the finite pixel weightings from the measurement data. This process recasts our integral equation as a linear inverse problem:

$$
\mathbf{A x}+\boldsymbol{\beta}=\mathbf{b},
$$

Where $\mathbf{A}$ is a coefficient matrix such that $A_{i j}$ is the length of the $j^{\text {th }}$ beam through the $i^{\text {th }}$ pixel, $\mathbf{x}=\left[x_{1}, x_{2}, \ldots\right]^{T}$ are the discretized (unknown) pixel weightings and $\mathbf{b}=$ are the measured data (the LHS of equation 1) contaminated by measurement noise $\boldsymbol{\beta}$, which is also unknown.

Solving equation 3 is problematic for a number of reasons. Firstly, unless the measurement noise $\boldsymbol{\beta}$ happens to lie in the column space of $\mathbf{A}$ there is no $\mathbf{x}$ which satisfies (3). We can instead search for a least-squares estimate $\mathbf{x}_{L S}$ in lieu of an exact match using:

$$
\mathbf{x}_{L S}=\min _{\mathbf{x}}|\mathbf{A x}-\mathbf{b}|_{2}^{2}
$$

But the resulting Euler equations:

$$
\mathbf{A}^{T} \mathbf{A x}=\mathbf{A}^{T} \mathbf{b}
$$

Lead to a singularity because $\mathbf{A}^{T} \mathbf{A}$ is rank-deficient and its inverse is undefined when the number of pixels is unequal to the number of beams (this is always the case in practical limited-data reconstruction).

\section{A. Landweber Iteration}

One commonly proposed solution $[14,15]$ is to take the gradient of $|\mathbf{A x}-\mathbf{b}|_{2}^{2}$ with respect to $\mathbf{x}$ and iteratively subtract a scalar multiple of it from an initial guess $\mathbf{x}_{0}=\mathbf{0}$. The result is a form of gradient descent called Landweber iteration:

$$
\mathbf{x}^{k+1}=\mathbf{x}^{k}+\alpha \mathbf{A}^{T}\left(\mathbf{A} \mathbf{x}^{k}-\mathbf{b}\right) .
$$

An added benefit to Landweber iteration is the ease with which prior knowledge can be incorporated into the solution method: we know the solution is smooth so we chose to apply a Gaussian filter to the solution between each iterative step as a way of repeatedly enforcing our prior knowledge.

\section{B. Tikhonov Inversion}

An alternative approach [16-18] is to define the prior knowledge in the initial optimization objective function. In this case, equation (4) is modified to include a penalty term $|\mathbf{L x}|$. When $\mathbf{L}$ is chosen to equal a discrete Laplacian, the new objective function penalizes large gradients in the image:

$$
\mathbf{x}_{\lambda}=\min _{\mathbf{x}}\left(|\mathbf{A x}-\mathbf{b}|_{2}^{2}+\lambda|\mathbf{L} \mathbf{x}|_{2}^{2}\right)
$$

The corresponding Euler equations for this new objective function are:

$$
\left(\mathbf{A}^{T} \mathbf{A}+\lambda \mathbf{L}^{T} \mathbf{L}\right) \mathbf{x}=\mathbf{A}^{T} \mathbf{b},
$$

Provided $\mathbf{L}$ is chosen with care, equation 7 can be solved directly:

$$
\mathbf{x}_{\lambda}=\left(\mathbf{A}^{T} \mathbf{A}+\lambda \mathbf{L}^{T} \mathbf{L}\right)^{-1} \mathbf{A}^{T} \mathbf{b} \equiv \mathbf{A}^{\#} \mathbf{b}
$$

These two methods are of a comparable speed for limited data reconstructions where the number of pixels does not exceed 1,000 and the number of beams does not exceed 100 . However, as the dimension of the problem increases the Tikhonov inversion suffers due to the relative increase in computation power required to invert matrices of larger sizes.

\section{BEAM OPTIMISATION}

One of the most challenging aspects of the turbine environment is the optical access, and a limitation of the number of beams (we can only use so many boreholes in the outer casing) and beam orientations (no beams can intersect the central axial driveshaft) leads us to a complicated problem of how to orient the beams within the measurement space. This question has recently been addressed [19] using the concept of model resolution matrices [20].

\section{A. Model resolution matrix}

We begin by substituting equation 3 into equation 8 :

$$
\mathbf{x}_{\lambda}=\mathbf{A}^{\#}(\mathbf{A x}+\boldsymbol{\beta}) .
$$

This gives us the Tikhonov inverse solution $\mathbf{x}_{\lambda}$ as a function of the true solution $\mathbf{x}$ and the measurement noise $\boldsymbol{\beta} . \mathbf{A}^{\#} \mathbf{A}$ is known as the model resolution matrix. If we consider that the error in the solution is probably dominated by a lack of measurement data as opposed to measurement noise (which is likely to be true with an estimated $28 \mathrm{~dB}$ signal-to-noise ratio typical of diode laser absorption measurements [21]), we can neglect the measurement noise term. Subtracting $\mathbf{x}$ from both sides and taking the norm gives an approximation of the Tikhonov error:

$$
\left|\mathbf{x}_{\lambda}-\mathbf{x}\right|_{2}^{2}=\left|\left(\mathbf{A}^{\#} \mathbf{A}-\mathbf{I}\right) \mathbf{x}\right|_{2}^{2}
$$

And dividing by the norm of the true solution gives us an expression for the maximum possible relative root-meansquared error (RMSE) of the reconstruction: 


$$
\begin{aligned}
& \left|\mathbf{x}_{\lambda}-\mathbf{x}\right|_{2}^{2} /|\mathbf{x}|_{2}^{2}=\left|\left(\mathbf{A}^{\#} \mathbf{A}-\mathbf{I}\right) \mathbf{x}\right|_{2}^{2} /|\mathbf{x}|_{2}^{2} ; \\
& \max _{\mathbf{x} \neq \mathbf{0}}\left|\mathbf{x}_{\lambda}-\mathbf{x}\right|_{2}^{2} /|\mathbf{x}|_{2}^{2} \equiv\left\|\mathbf{A}^{\#} \mathbf{A}-\mathbf{I}\right\|_{2},
\end{aligned}
$$

Where $\left\|\mathbf{A}^{\#} \mathbf{A}-\mathbf{I}\right\|_{2}$ is the spectral norm of $\mathbf{A}^{\#} \mathbf{A}-\mathbf{I}$. By minimising this value we can minimize the upper limit on the relative RMSE of the Tikhonov inverse solution (in a noisefree simulation), and this is the objective of our global optimization approach. This approach is inspired by the recent advances in beam optimization from Twynstra and Daun [19], although our own objective function and genetic algorithm are slightly modified.

\section{B. Genetic algorithm}

A genetic algorithm was used to find an approximate global minimum of the RHS in equation 12 for a particular annular geometry $0.5<r<1, \phi=[0,2 \pi)$ to represent a cross section of the turbine engine which is perpendicular to the engine axis. The iteration was initiated with 2,000 randomly generated beam configurations from which the top 50 (with lowest spectral norm) were selected and used to seed the second generation. For each selected beam configuration 40 "child" configurations were generated by randomly perturbing the beam start/end points with Gaussian noise with a variance that was decreased as the iteration progressed. Figure 1 shows the progress of the first optimization attempt.

This irregular configuration was tested against a set of regular fan-beam configurations, some of which are shown in figure 2. Randomized temperature and mole-fraction phantoms were generated with constant smoothness, and from these phantoms transmittance data were generated using a spectrum model and HITRAN database parameters [7]. Gaussian noise was added to the synthetic data to a level of

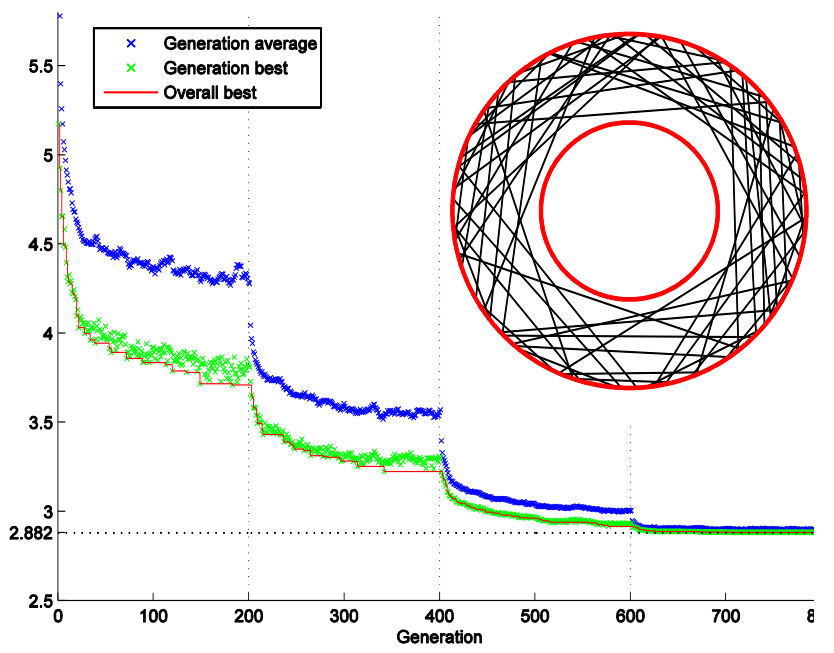

Figure 1. Progress of a genetic algorithm to optimize beam configuration. Every 200 generations the perturbation noise was reduced (vertical dotted lines). The resulting beam configuration after 800 iterations is shown in the top right corner.

$28 \mathrm{~dB}$ signal-to-noise ratio, and the reconstructions were performed using both Landweber and Tikhonov solver algorithms.
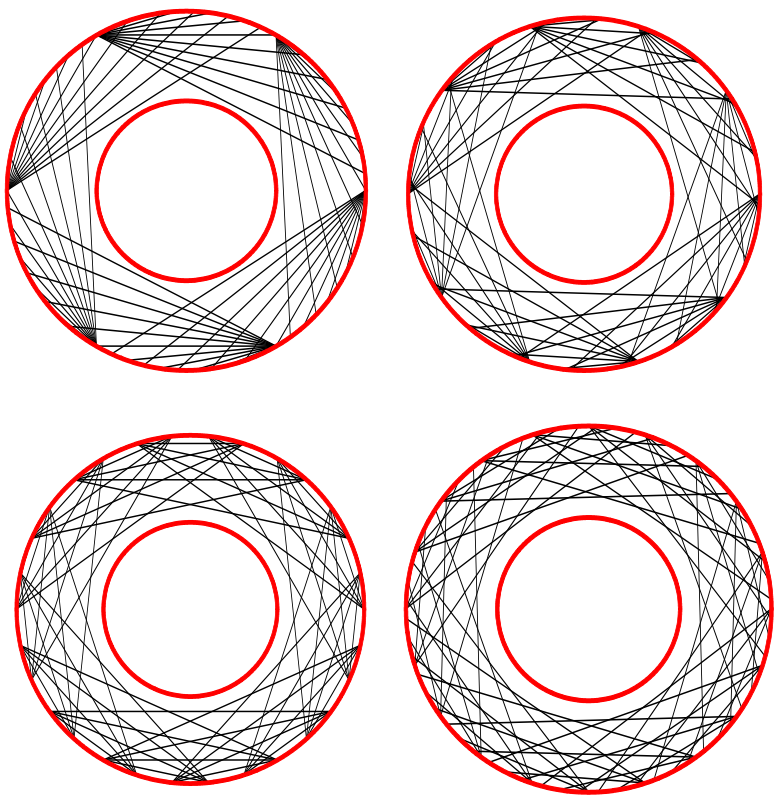

Figure 2. Regular fan-beam configurations. Clockwise, from top-left: 6 sources, 10 sources, 15 sources and 20 sources. All configurations in this study are of 60 beams.

\section{RESULTS}

For each beam configuration, the synthetic data generation and reconstructions were repeated 24 times. The average temperature error (\% RMSE) over all 24 reconstructions is plotted for each beam configuration and reconstruction method in figure 3. 'irreg501' refers to the beam configuration seen in figure 1, and 'irreg502' is a second optimized configuration generated by repeating the genetic algorithm with a new initial starting point: the algorithm has clearly converged on two different configurations, and we expect there to exist many more similarly successful approaches.

The results show that the irregular beam configurations both outperform regular fan-beam arrangements in the annular geometry when Tikhonov inversion is used, and are of a comparable accuracy when Landweber iteration is used. The fan-beam configuration with 6 sources is the worst of the set with around 3-3.2 \% temperature error (roughly $50 \mathrm{~K}$ absolute), whilst the first irregular configuration shown in figure 1 is the best with errors of only $2.5-2.6 \%$.

\section{CONCLUSIONS}

The numerical simulations above demonstrate the feasibility of beam optimization for laser absorption tomography in geometrically constrained environments where a limited number of beams is a considerable design issue for any imaging sensor. Convergence of the genetic solver can be achieved on a desktop PC within a matter of hours, and the resulting optimized beam configurations are shown to outperform conventional fan-beam arrangements when tested on a range of 24 different randomly generated temperature phantoms. 


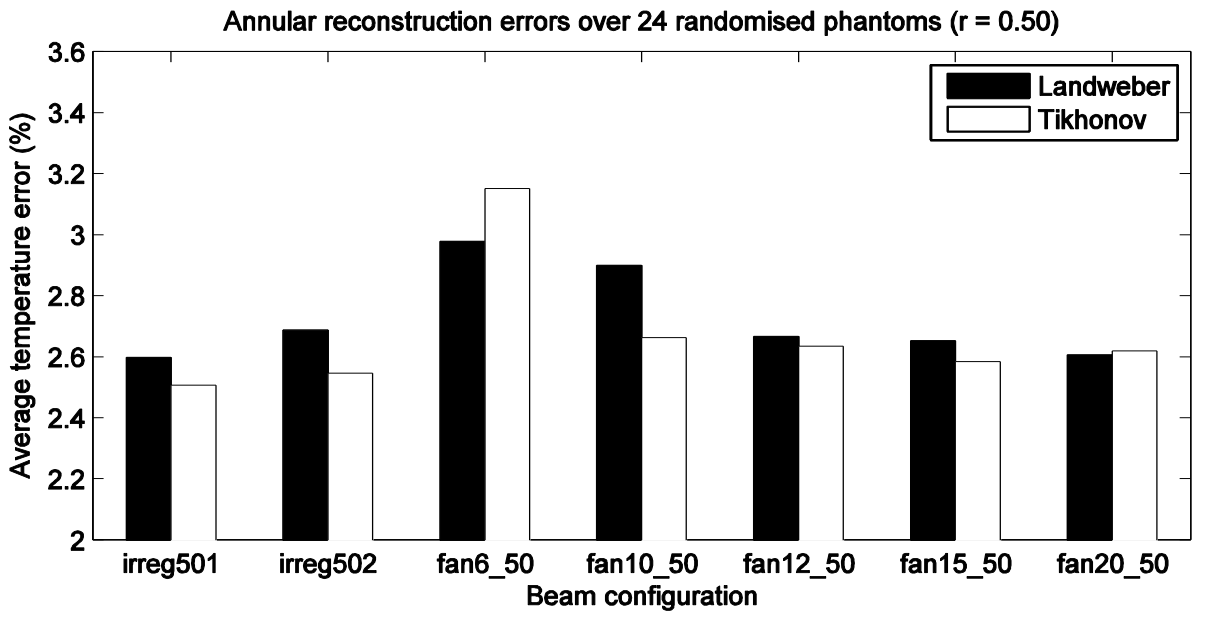

Figure 3. Temperature reconstruction accuracies averaged over 24 independent simulated experiments for randomly generated gas temperature and species concentration distributions. 'fan6_50' to 'fan20_50' represent regular fan-beam configurations with 6 to 20 sources ('fan12_50' is omitted from figure 2).

\section{ACKNOWLEDGEMENT}

The authors wish to thank M. Twynstra and K. Daun for their invaluable comments and preceding work which has motivated this study.

\section{REFERENCES}

[1] K. Clive and I. Paul, "An overview of the measurement errors associated with gas turbine aeroengine pyrometer systems," Measurement Science and Technology, vol. 13, p. 873, 2002.

[2] C. Kerr and P. Ivey, "Optical pyrometry for gas turbine aeroengines," Sensor Review, vol. 24, pp. 378 - 3862004.

[3] P. M. Brisley, et al., "Three-dimensional temperature measurement of combustion flames using a single monochromatic CCD camera," Instrumentation and Measurement, IEEE Transactions on, vol. 54, pp. 1417-1421, 2005.

[4] M. P. Lee, et al., "Temperature measurements in gases by use of planar laser-induced fluorescence imaging of NO," Applied Optics, vol. 32, pp. 5379-5396, 1993.

[5] R. K. Hanson and P. K. Falcone, "Temperature measurement technique for high-temperature gases using a tunable diode laser," Appl. Opt., vol. 17, pp. 2477-2480, 1978.

[6] M. P. Arroyo and R. K. Hanson, "Absorption measurements of water-vapor concentration, temperature, and line-shape parameters using a tunable InGaAsP diode laser," Appl. Opt., vol. 32, pp. 6104-6116, 1993

[7] L. S. Rothman, et al., "The HITRAN 2008 molecular spectroscopic database," Journal of Quantitative Spectroscopy and Radiative Transfer, vol. 110, pp. 533-572, 2009/7// 2008.

[8] M. P. Wood and K. B. Ozanyan, "Concentration and Temperature Tomography at Elevated Pressures," Sensors Journal, IEEE, vol. PP, pp. 1-1, 2013.

[9] V. L. Kasyutich and P. A. Martin, "Towards a two-dimensional concentration and temperature laser absorption tomography sensor system," Applied Physics B, vol. 102, pp. 149-162, 2011/01/01 2011.

[10] J. Song, et al., "Algebraic tomographic reconstruction of twodimensional gas temperature based on tunable diode laser absorption spectroscopy," Applied Physics B, pp. 1-9, 2013/04/08 2013.
L. Ma, et al., "50-kHz-rate 2D imaging of temperature and $\mathrm{H} 2 \mathrm{O}$ concentration at the exhaust plane of a J85 engine using hyperspectral tomography," Opt. Express, vol. 21, pp. 1152-1162, 2013.

[12] F. Wang and et al., "Two-dimensional tomography for gas concentration and temperature distributions based on tunable diode laser absorption spectroscopy," Measurement Science and Technology, vol. 21, p. 045301, 2010.

[13] V. Nagali and R. K. Hanson, "Design of a diode-laser sensor to monitor water vapor in high-pressure combustion gases," Appl. Opt., vol. 36, pp. 9518-9527, 1997.

[14] L. Landweber, "An Iteration Formula for Fredholm Integral Equations of the First Kind," American Journal of Mathematics, vol. 73, pp. 615-624, 1951.

[15] V. M. Fridman, "The method of successive approximations for a Fredholm integral equation of the first kind," Uspehi Mat. Mauk., vol. 11, pp. 223-224, 1956.

[16] D. L. Phillips, "A Technique for the Numerical Solution of Certain Integral Equations of the First Kind," J. ACM, vol. 9, pp. 84-97, 1962.

[17] A. Tikhonov, "Solution of incorrectly formulated problems and the regularization method," in Soviet Math. Doklady, 1963, pp. 10351038.

[18] S. Twomey, "On the Numerical Solution of Fredholm Integral Equations of the First Kind by the Inversion of the Linear System Produced by Quadrature," J. ACM, vol. 10, pp. 97-101, 1963.

[19] M. G. Twynstra and K. J. Daun, "Laser-absorption tomography beam arrangement optimization using resolution matrices," Appl. Opt., vol. 51, pp. 7059-7068, 2012.

[20] R. A. Wiggins, "The general linear inverse problem: Implication of surface waves and free oscillations for Earth structure," Reviews of Geophysics, vol. 10, pp. 251-285, 1972.

[21] F. P. Hindle, et al., "Measurement of gaseous hydrocarbon distribution by a near-infrared absorption tomography system," Journal of Electronic Imaging, vol. 10, pp. 593-600, 2001. 